\title{
Massively parallel computation of large-scale spatial price equilibrium models with discriminatory ad valorem tariffs
}

\author{
Anna Nagurney \\ School of Management, University of Massachusetts, \\ Amherst, MA 01003, USA \\ Charles F. Nicholson and Phillip M. Bishop \\ Department of Agricultural, Resource and Managerial Economics, \\ Cornell University, Ithaca, NY 14853, USA
}

\begin{abstract}
In this paper, we develop a perfectly competitive spatial equilibrium model in price and quantity variables in the presence of discriminatory ad valorem tariffs, a widely used trade policy instrument. We derive the equilibrium conditions and formulate them as a variational inequality problem. An algorithm is then proposed for the computation of the equilibrium pattern and convergence results established. The algorithm resolves the problem into very simple subproblems, each of which can be solved simultaneously and in closed form. Finally, the algorithm is implemented on the massively parallel Thinking Machines CM-2 and CM-5 architectures, known as the Connection Machines, and numerical results presented.
\end{abstract}

Keywords: Spatial price equilibria, discriminatory ad valorem tariffs, variational inequalities, massively parallel computation.

\section{Introduction}

A principal issue in the study of spatial price equilibrium problems is the computation of the commodity production, consumption, and trade patterns in spatially separated markets, as well as the commodity prices. Such models, which date to Samuelson [15] and Takayama and Judge [18], have provided a basic framework for the modeling of trade in agricultural and energy markets. In addition, they have been the focus of both policy modeling extensions, as well as the development of a variety of algorithmic schemes, because the problems encountered in practice are typically large scale. 
Early rigorous computational approaches (cf. Takayama and Judge [17,18]) were based, under appropriate assumptions, on reformulations of the problems as optimization problems, in which case the governing equilibrium conditions were equivalent to the Kuhn-Tucker conditions. In particular, symmetry of the Jacobians of the economic functions was required which precluded realistic modeling of asymmetric interactions in the functions needed, for example, in studying multicommodity problems. More recent treatments have included using complementarity theory and variational inequality theory for algorithm development. For background and extensive references, we refer the reader to Thore [21] and Nagurney [9].

Extensions of spatial price equilibrium models to include a plethora of policies using a variety of mathematical methodologies have been made by, among others, Thore [20], Thompson and Thore [19], Nagurney and Zhao [13], Thore et al. [22], and Nagurney et al. [12]. Such extensions have included rigid prices, price supports in the form of price floors on the production side and price ceilings on the consumption side, and goal targets.

In this paper, we consider the formulation and computation of spatial price equilibrium problems with discriminatory ad valorem tariffs. The challenge faced in formulating and solving spatial price equilibrium problems with discriminatory ad valorem tariffs is that in their presence, the equilibrium conditions can no longer be reformulated as an optimization problem, even in the simplest case of linear supply and demand functions and fixed transaction costs. This characteristic was recognized as early as Takayama and Judge [18].

In particular, building upon the work of Takayama and Judge [18], we develop a model with ad valorem tariffs in which supply and demand functions, rather than their inverses, are assumed known, and with unit transaction costs, which we assume include the unit transportation costs. Spatial price equilibrium models with both price and quantity variables have received less attention in the literature than quantity models in which the supply and demand price functions are assumed known. The mode of behavior assumed here is that of price-taking, that is, we assume perfect competition. Moreover, we assume that the commodity produced is homogeneous.

The motivation for this research stems, in part, from the need to establish mathematical frameworks for this class of trade policy instruments, which is used by nearly all nations to restrict imports of a wide variety of commodities.

The paper is organized as follows. In section 2, we introduce the perfectly competitive spatial price equilibrium model with ad valorem tariffs and relate it to other such models in the literature. We state the equilibrium conditions and then derive the governing variational inequality formulation.

In section 3, we propose the modified projection method of Korpelevich [8] for the computation of the equilibrium supply price, demand price, and commodity trade pattern. We then prove conditions for convergence of the algorithm. The notable feature of the algorithm is that its realization in the context of our model yields subproblems of special structure which can then be solved simultaneously and in closed form. 
It is this feature that we then exploit in section 4, where we discuss the implementation of the algorithm on two massively parallel architectures, the Thinking Machines CM-2 and CM-5, also known as the Connection Machines. Finally, in this section we present numerical results to illustrate the performance of the algorithm for the computation of solutions to the new model on these advanced architectures.

In section 5, we summarize our results and present our conclusions.

\section{The spatial equilibrium model in price and quantity variables with discriminatory ad valorem tariffs}

In this section, we develop the perfectly competitive spatial market model with ad valorem tariffs. The model assumes that a single homogeneous commodity is produced and consumed. It also assumes that the supply and demand price functions are given as well as the unit transaction cost functions, which are assumed to incorporate the unit transportation costs. The governing equilibrium conditions are stated and the equilibrium solution is then shown to satisfy a variational inequality problem.

We consider $m$ supply markets involved in the production of the homogeneous commodity and $n$ demand markets. We denote a typical supply market by $i$ and a typical demand market by $j$. Let $s_{i}$ denote the supply at supply market $i$ and $d_{j}$ the demand at demand market $j$. We group the supplies into a row vector $s \in \mathbb{R}^{m}$ and the demands into a row vector $d \in \mathbb{R}^{n}$. Let $Q_{i j}$ denote the nonnegative commodity shipment between supply and demand market pair $(i, j)$, and group the commodity shipments into a column vector $Q \in \mathbb{R}^{m n}$.

We now describe the price and cost structure. Let $\pi_{i}$ denote the supply price at supply market $i$ and $\rho_{j}$ the demand price at demand market $j$. We group the supply prices into a column vector $\pi \in \mathbb{R}^{m}$ and the demand prices into a column vector $\rho \in \mathbb{R}^{n}$. The unit transaction cost associated with shipping/trading the commodity between supply market $i$ and demand market $j$ is denoted by $c_{i j}$. We group the transaction costs into a row vector $c \in \mathbb{R}^{m n}$.

We assume that the supply at a supply market may, in general, depend upon the supply price of the commodity at every supply market, that is,

$$
s=s(\pi) .
$$

Similarly, the demand at a demand market may, in general, depend upon the demand prices of the commodity at every demand market, that is,

$$
d=d(\rho) .
$$

The per unit transaction cost, in turn, associated with shipping the commodity between a pair of supply and demand markets is assumed to be, in general, a function of the entire commodity shipment pattern, that is,

$$
c=c(Q) \text {. }
$$


In the absence of policy interventions, and under the assumption of perfect competition, the well-known spatial price equilibrium conditions (cf. Takayama and Judge $[17,18])$ are stated as follows: A commodity supply price, shipment, and demand price pattern $\left(\pi^{*}, Q^{*}, \rho^{*}\right)$ is in equilibrium if it satisfies the following systems of equalities and inequalities. For all supply markets $i, i=1, \ldots, m$ :

$$
s_{i}\left(\pi^{*}\right) \begin{cases}=\sum_{j} Q_{i j}^{*}, & \text { if } \pi_{i}^{*}>0, \\ \geq \sum_{j} Q_{i j}^{*}, & \text { if } \pi_{i}^{*}=0\end{cases}
$$

for all pairs of supply and demand markets $(i, j), i=1, \ldots, m ; j=1, \ldots, n$ :

$$
\pi_{i}^{*}+c_{i j}\left(Q^{*}\right) \begin{cases}=\rho_{j}^{*}, & \text { if } Q_{i j}^{*}>0, \\ \geq \rho_{j}^{*}, & \text { if } Q_{i j}^{*}=0,\end{cases}
$$

and for all demand markets $j, j=1, \ldots, n$ :

$$
d_{j}\left(\rho^{*}\right) \begin{cases}=\sum_{i} Q_{i j}^{*}, & \text { if } \rho_{j}^{*}>0, \\ \leq \sum_{i} Q_{i j}^{*}, & \text { if } \rho_{j}^{*}=0 .\end{cases}
$$

In other words, in equilibrium, if the supply price of the commodity at a supply market is positive, then the supply is equal to the sum of the commodity shipments out of that market; if the supply price is zero, then the supply can exceed the commodity shipments out of that market. Also, if a positive amount of commodity is shipped between a pair of supply and demand markets, then the supply price at the supply market plus the cost of transaction must be equal to the demand price at the demand market. There will be no shipment of the commodity between a pair of markets if the sum of the supply price and transaction cost exceeds the demand price. Finally, if the demand price of the commodity is positive at a demand market, then the demand is equal to the commodity shipments to that demand market; if the demand price is zero, then the commodity shipments into that demand market can exceed the demand.

We now introduce discriminatory ad valorem tariffs into the above model. Let $\tau_{i j}$ denote the ad valorem tariff, assumed positive and finite, and applied to imports by demand market $j$ from supply market $i$. The incorporation of ad valorem tariffs modifies the spatial price equilibrium conditions as follows. Conditions (4) and (6) remain as before, whereas condition (5) is now modified to: For all pairs of supply and demand markets $(i, j), i=1, \ldots, m ; j=1, \ldots, n$ :

$$
\left(\pi_{i}^{*}+c_{i j}\left(Q^{*}\right)\right) \cdot\left(1+\tau_{i j}\right) \begin{cases}=\rho_{j}^{*}, & \text { if } Q_{i j}^{*}>0, \\ \geq \rho_{j}^{*}, & \text { if } Q_{i j}^{*}=0 .\end{cases}
$$


Hence, in equilibrium, if a positive amount of the commodity is shipped between a pair of supply and demand markets, then the effective supply price plus transaction cost after the imposition of ad valorem tariffs must be equal to the demand price at the demand market. If there is no commodity shipment between a pair of supply and demand markets, then the effective supply price plus transaction cost can exceed the demand price.

As has been mentioned in section 1 , quantity models in the spatial price equilibrium framework, in which it is assumed that supply and demand price functions are given, rather than the supply and demand functions as considered here, have received more attention in the literature than models with price and quantity variables. This may be due, in part, to the greater simplicity of the underlying network structure of the models as well as to the reduced number of variable which make the quantity model easier to handle from an algorithmic development standpoint. A similar situation arises when one incorporates ad valorem tariffs.

Before deriving the variational inequality formulation of equilibrium conditions (4), (7), and (6), we briefly highlight other spatial market models with tariffs in the literature. Non-discriminatory ad valorem tariffs, that is, ad valorem tariffs that do not vary by exporting country, have been incorporated into a spatial price equilibrium framework by modification of linear and separable excess supply and demand price functions in a quadratic programming framework by Cramer et al. [3]. Holland and Sharples [7], in turn, proposed a fixed-point algorithm to compute solutions to spatial price models with ad valorem tariffs, whereas Chang et al. [2] proposed a heuristic method. More recently, Nagurney et al. [10] considered the quantity spatial price equilibrium model with ad valorem tariffs in a variational inequality framework. This model, unlike many earlier models, was not limited to linear and symmetric functions.

We now derive the variational inequality formulation of the equilibrium conditions governing the spatial market model with ad valorem tariffs. In particular, we state the following.

Theorem 1 A supply price, shipment, and demand price pattern $\left(\pi^{*}, Q^{*}, \rho^{*}\right) \in \mathbb{R}_{+}^{m+m n+n}$ is an equilibrium pattern in the presence of ad valorem tariffs, that is, satisfies conditions (4), (7), and (6), if and only if it satisfies the variational inequality problem

$$
\begin{aligned}
& \sum_{i=1}^{m}\left(s_{i}\left(\pi^{*}\right)-\sum_{j=1}^{n} Q_{i j}^{*}\right) \cdot\left(\pi_{i}-\pi_{i}^{*}\right) \\
& \left.\quad+\sum_{i=1}^{m} \sum_{j=1}^{n}\left(\left(\pi_{i}^{*}+c_{i j}\left(Q^{*}\right)\right)\left(1+\tau_{i j}\right)-\rho_{j}^{*}\right)\right) \cdot\left(Q_{i j}-Q_{i j}^{*}\right) \\
& \quad-\sum_{j=1}^{n}\left(d_{j}\left(\rho^{*}\right)-\sum_{i=1}^{m} Q_{i j}^{*}\right) \cdot\left(\rho_{j}-\rho_{j}^{*}\right) \geq 0, \quad \forall(\pi, Q, \rho) \in \mathbb{R}_{+}^{m+m n+n} .
\end{aligned}
$$


Proof We first establish that if a pattern $\left(\pi^{*}, Q^{*}, \rho^{*}\right)$ satisfies equilibrium conditions (4), (7), and (6), then it also satisfies variational inequality (8).

For fixed supply market $i$, (4) implies that

$$
\left(s_{i}\left(\pi^{*}\right)-\sum_{j} Q_{i j}^{*}\right) \cdot\left(\pi_{i}-\pi_{i}^{*}\right) \geq 0, \quad \forall \pi_{i} \geq 0 .
$$

For fixed market pair $(i, j),(7)$ implies that

$$
\left(\left(\pi_{i}^{*}+c_{i j}\left(Q^{*}\right)\right) \cdot\left(1+\tau_{i j}\right)-\rho_{j}^{*}\right) \cdot\left(Q_{i j}-Q_{i j}^{*}\right) \geq 0, \quad \forall Q_{i j} \geq 0,
$$

whereas for fixed demand market $j$, (6) implies that

$$
-\sum_{j}\left(d_{j}\left(\rho^{*}\right)-\sum_{i} Q_{i j}^{*}\right) \cdot\left(\rho_{j}-\rho_{j}^{*}\right) \geq 0, \quad \forall \rho_{j} \geq 0 .
$$

Summing now (9) over all supply markets $i,(10)$ over all pairs of markets $(i, j)$, and (11) over all demand markets $j$, we obtain variational inequality (8).

We now show that any solution to variational inequality (8) also satisfies equilibrium conditions (4), (7), and (6).

In variational inequality (8), set $\pi_{i}=\pi_{i}^{*}$ for all $i \neq k, Q_{i j}=Q_{i j}^{*}$ for all $(i, j)$, and $\rho_{j}=\rho_{j}^{*}$ for all $j$. Then $(8)$ reduces to

$$
\left(s_{k}\left(\pi^{*}\right)-\sum_{j} Q_{k j}^{*}\right) \cdot\left(\pi_{k}-\pi_{k}^{*}\right) \geq 0, \quad \forall \pi_{k} \geq 0,
$$

which implies equilibrium condition (4) for supply market $k$. Similarly, in variational inequality (8), set $Q_{i j}=Q_{i j}^{*}$ for all market pairs $(i, j) \neq(k, l)$. Then (8) reduces to:

$$
\left(\left(\pi_{k}^{*}+c_{k l}\left(Q^{*}\right)\right)-\rho_{l}^{*}\right) \cdot\left(1+\tau_{k l}\right) \cdot\left(Q_{k l}-Q_{k l}^{*}\right) \geq 0, \quad \forall Q_{k l} \geq 0,
$$

which implies equilibrium conditions (7) for market pair $(k, l)$.

Similar arguments can be used on the demand side to establish equilibrium condition (6).

Because the above statements are independent of how we select the markets, the conditions hold for all markets and market pairs.

In the absence of discriminatory ad valorem tariffs, the variational inequality (8) collapses to the variational inequality problem formulation of equilibrium conditions (4), (5), and (6) derived in Dafermos and McKelvey [6] (see also Nagurney [9]) and given by 


$$
\begin{gathered}
\sum_{i=1}^{m}\left(s_{i}\left(\pi^{*}\right)-\sum_{j=1}^{n} Q_{i j}^{*}\right) \cdot\left(\pi_{i}-\pi_{i}^{*}\right)+\sum_{i=1}^{m} \sum_{j=1}^{n}\left(\pi_{i}^{*}+c_{i j}\left(Q^{*}\right)-\rho_{j}^{*}\right) \cdot\left(Q_{i j}-Q_{i j}^{*}\right) \\
-\sum_{j=1}^{n}\left(d_{j}\left(\rho^{*}\right)-\sum_{i=1}^{m} Q_{i j}^{*}\right) \cdot\left(\rho_{j}-\rho_{j}^{*}\right) \geq 0, \quad \forall(\pi, Q, \rho) \in \mathbb{R}_{+}^{m+m n+n} .
\end{gathered}
$$

Takayama and Judge [18] proposed a linear complementarity formulation of the spatial price equilibrium conditions in the presence of discriminatory ad valorem tariffs for a model with linear supply and demand price functions. Indeed, even in the simplest case of linear and separable supply and demand price functions, the model with tariffs (unlike the classical spatial price equilibrium model) could not be reformulated as a quadratic programming problem.

Variational inequality (8) can be put into standard form (cf. Nagurney [9]), that is:

Determine $X^{*} \in K$, such that

$$
F\left(X^{*}\right) \cdot\left(X-X^{*}\right) \geq 0, \quad \forall X \in K,
$$

where $K$ is the feasible set and is assumed to be closed and convex.

Indeed, let $X \equiv(\pi, Q, \rho)$ and let $F \equiv\left(F^{1}, F^{2}, F^{3}\right)$, where the components of $F^{1}$ are

the components of $F^{2}$ are

$$
F_{i}^{1}(\pi, Q, \rho) \equiv s_{i}(\pi)-\sum_{j} Q_{i j}, \quad \text { for } i=1, \ldots, m,
$$

$$
F_{i j}^{2}(\pi, Q, \rho) \equiv\left(\pi_{i}+c_{i j}(Q)\right) \cdot\left(1+\tau_{i j}\right)-\rho_{j}, \quad \text { for } i=1, \ldots, m ; j=1, \ldots, n,
$$

and the components of $F^{3}$ are

$$
F_{j}^{3}(\pi, Q, \rho) \equiv-d_{j}(\rho)+\sum_{i} Q_{i j}, \quad \text { for } j=1, \ldots, n,
$$

with the feasible set $K \equiv \mathbb{R}_{+}^{m+m n+n}$.

Moreover, we note that, since $K$ is a cone, variational inequality (8), equivalently, (15), is equivalent to the complementarity problem:

Determine $X^{*} \in \mathbb{R}_{+}^{m+m n+n}$, such that

$$
F\left(X^{*}\right)^{T} \geq 0, \quad X^{*} \geq 0 \text { and } F\left(X^{*}\right) \cdot X^{*}=0 .
$$

We now discuss certain qualitative properties of the function $F(\pi, Q, \rho)$ that will be useful in establishing convergence of the computational procedure. In particular, we give conditions that guarantee Lipschitz continuity of $F$ and then monotonicity.

Recalling that the feasible set $K$ is convex here, and invoking the Mean Value Theorem, we immediately obtain 
Lemma $1 F(X)$ is Lipschitz continuous on $K$, that is, there exists an $L>0$ such that

$$
\left\|F\left(X^{1}\right)-F\left(X^{2}\right)\right\| \leq L\left\|X^{1}-X^{2}\right\|, \quad \forall X^{1}, X^{2} \in K,
$$

under the assumption that the components of $F$ have bounded first-order derivatives for all $X \in K$.

It follows that $F(X)$ will have bounded first-order derivatives if the supply and demand functions and the unit transaction cost functions have bounded first-order derivatives and the tariff rates are all finite.

We now present a lemma that will be used to establish monotonicity of $F$. Recall that $F(X)$ is said to be monotone if

$$
\left[F\left(X^{1}\right)-F\left(X^{2}\right)\right] \cdot\left[X^{1}-X^{2}\right] \geq 0, \quad \forall X^{1}, X^{2} \in K .
$$

Since the lemma is just a statement of the Schur complement determinant formula, it is provided without proof (cf. Strang [16, p. 222]).

Lemma 2 If a matrix $A$ is square and nonsingular, and $w^{T}$ and $z$ are two vectors, with 0 denoting the scalar zero, and $|B|$ denoting the determinant of $B$, then

$$
\left|\left(\begin{array}{cc}
A & z \\
w^{T} & 0
\end{array}\right)\right|=\left|\left(-w^{T} A^{-1} z\right) \| A\right| .
$$

Lemma 3 Assume that $s(\pi), c(Q)$, and $-\rho(d)$ are each strongly monotone, respectively, in $\pi, Q$, and $\rho$, that is,

$$
\begin{array}{cc}
{\left[s\left(\pi^{1}\right)-s\left(\pi^{2}\right)\right] \cdot\left[\pi^{1}-\pi^{2}\right] \geq \alpha\left\|\pi^{1}-\pi^{2}\right\|} & \forall \pi^{1}, \pi^{2} \in K, \\
{\left[c\left(Q^{1}\right)-c\left(Q^{2}\right)\right] \cdot\left[Q^{1}-Q^{2}\right] \geq \beta\left\|Q^{1}-Q^{2}\right\|,} & \forall Q^{1}, Q^{2} \in K, \\
-\left[d\left(\rho^{1}\right)-d\left(\rho^{2}\right)\right] \cdot\left[\rho^{1}-\rho^{2}\right] \geq \mu\left\|\rho^{1}-\rho^{2}\right\|, & \forall \rho^{1}, \rho^{2} \in K,
\end{array}
$$

with $\alpha>0, \beta>0$, and $\mu>0$.

Then $F$ with components defined by (16), (17), and (18) is monotone.

Proof We use the fact that the Jacobian of $F, \nabla F$, is positive semidefinite over $K$ is and only if $F$ is monotone (over $K$ ) (Ortega and Rheinboldt [14, p. 142]). Also, we use the fact that a function is strongly monotone over the feasible set $K$ if and only if its Jacobian is positive definite over $K$ (see, e.g., Dafermos [4, appendix 1]). In particular, we establish that the Jacobian of $F$ can be expressed as the sum of two matrices, each of which, under the imposed assumptions, is positive semidefinite. 
Note that

where

$$
\nabla F(X)=B+C,
$$

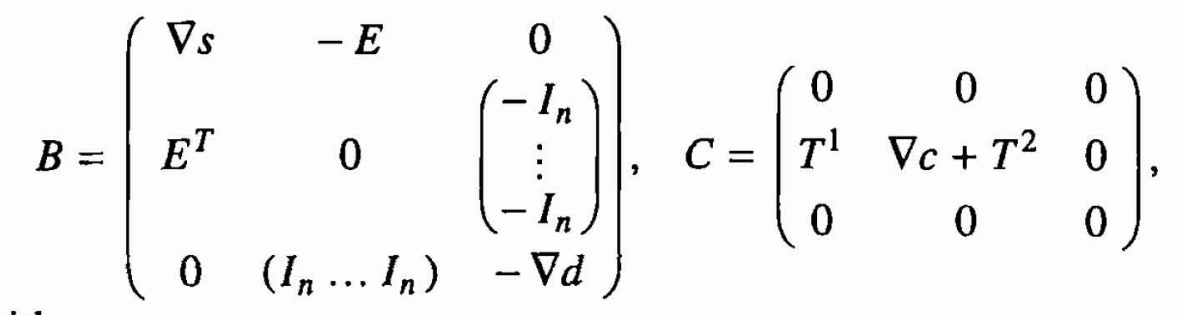

with

$$
\begin{aligned}
E & =\left(\begin{array}{cccc}
e_{1} & & & \\
& e_{1} & & \\
& & \ddots & \\
& & & e_{1}
\end{array}\right) \text { and } e_{1}=[1, \ldots, 1]_{1 \times n}, \\
T^{1} & =\left(\begin{array}{cccc}
\tau_{1} & & & \\
& \tau_{2} & & \\
& & \ddots & \\
& & & \tau_{m}
\end{array}\right), T^{2}=\left(\begin{array}{ccc}
\tau_{1} & \cdots & \tau_{1} \\
\tau_{2} & \cdots & \tau_{2} \\
\vdots & & \vdots \\
\tau_{m} & & \tau_{m}
\end{array}\right) \text { and } \tau_{i}=\left[\tau_{i 1}, \tau_{i 2}, \ldots, \tau_{i n}\right]_{n \times 1}
\end{aligned}
$$

Without any loss of generality, we consider matrix $\bar{B}$, which corresponds to a simple transformation of matrix $B$ without any change in properties, and we show that $\bar{B}$ is positive semidefinite, where

$$
\bar{B}=\left(\begin{array}{ccc}
\nabla s & 0 & -E \\
0 & -\nabla d & \left(I_{n} \ldots I_{n}\right) \\
E^{T} & \left(\begin{array}{c}
-I_{n} \\
\vdots \\
-I_{n}
\end{array}\right) & 0
\end{array}\right) .
$$

In particular, the matrices $\bar{B}$ and $B$ are related via the permutation matrix $P$ such that $P \bar{B} P^{T}=B$, where

$$
P=\left(\begin{array}{lll}
I & 0 & 0 \\
0 & 0 & I \\
0 & I & 0
\end{array}\right),
$$

Consider all the principal minors of $\bar{B}$ :

$$
\left|\bar{B}_{1}\right|,\left|\bar{B}_{2}\right|, \ldots,\left|\bar{B}_{m}\right|, \ldots,\left|\bar{B}_{m+n}\right|,\left|\bar{B}_{m+n+1}\right|, \ldots,\left|\bar{B}_{m+n+m n}\right| \text {. }
$$


Since under the assumption that $s$ and $-d$ are strongly monotone and, hence, $\nabla s$ and $\nabla d$ are positive definite, it follows that $\left|\bar{B}_{1}\right|, \ldots,\left|\bar{B}_{m+n}\right|$ are all positive. Now consider $\left|\bar{B}_{m+n+1}\right|$, which is of the form

where here, specifically, $w^{T}=-z^{T}$.

$$
\left|\bar{B}_{m+n+1}\right|=\left(\begin{array}{cc}
A & z \\
w^{T} & 0
\end{array}\right),
$$

Applying now lemma 2, we obtain

$$
\left|\bar{B}_{m+n+1}\right|=\left|\left(z^{T}\left(\begin{array}{cc}
\nabla s & 0 \\
0 & -\nabla d
\end{array}\right)^{-1} z\right)\right|\left|\left(\begin{array}{cc}
\nabla s & 0 \\
0 & -\nabla d
\end{array}\right)\right| \geq 0 .
$$

Hence, $\left|\bar{B}_{m+n+1}\right|$ is positive semidefinite. By induction, we can conclude that $\left|\bar{B}_{m+n+2}\right| \geq 0, \ldots,\left|\bar{B}_{m+n+m n}\right| \geq 0$, which implies that $\bar{B}$ is positive semidefinite and, hence, so is $B$.

Note that matrix $C$ can be rewritten as the sum of the following two matrices:

$$
C=\left(\begin{array}{ccc}
0 & 0 & 0 \\
T^{1} & \nabla c & 0 \\
0 & 0 & 0
\end{array}\right)+\left(\begin{array}{ccc}
0 & 0 & 0 \\
0 & T^{2} & 0 \\
0 & 0 & 0
\end{array}\right)
$$

with the second matrix in the sum consisting of identical rows in view of the definition of $T^{2}$ and, hence, its determinant is zero. Since the transaction cost functions are assumed to be strongly monotone, it further follows, using the same arguments as previously and lemma 2 , that the first matrix in this sum must also be positive semidefinite. We conclude that matrix $C$ is also positive semidefinite.

Again, using the fact that the sum of two positive semidefinite matrices is again positive semidefinite, we conclude that $\nabla F(X)$ is positive semidefinite and, hence, $F$ is monotone.

\section{The computational procedure}

In this section, we propose an algorithm for the computation of the solution of variational inequality (8) governing the spatial equilibrium model in price and quantity variables and with discriminatory ad valorem tariffs. We also establish convergence.

The algorithm that we propose is the modified projection method of Korpelevich [8] which, as we shall show, resolves the variational inequality problem under consideration here into subproblems that are computationally simple. Indeed, we obtain a closed-form expression for the determination of the supply prices, demand prices, and the commodity shipments at each iteration. Moreover, because each of the supply prices at a supply market, each of the demand prices at a demand market, and the commodity shipments between a pair of supply and demand markets can be 
evaluated separately and simultaneously at any iteration, this algorithmic scheme enables one to exploit the availability of (massively) parallel computer architectures.

We first present the general statement of the algorithm, motivate its realization in the context of the model, and then present its precise statement in that context, highlighting its special features.

\section{The modified projection method}

\section{Step 0: Initialization}

Start with an $X^{0} \in K$. Set $k:=1$ and select $\gamma$, such that $0<\gamma<1 / L$, where $L$ is the Lipschitz constant in (19).

\section{Step 1: Construction and computation}

Compute $\bar{X}^{k-1}$ by solving the variational inequality subproblem:

$$
\left[\bar{X}^{k-1}+\left(\gamma F\left(X^{k-1}\right)^{T}-X^{k-1}\right)\right]^{T} \cdot\left[X^{\prime}-\bar{X}^{k-1}\right] \geq 0, \quad \forall X^{\prime} \in K .
$$

\section{Step 2: Adaptation}

Compute $X^{k}$ by solving the variational inequality subproblem:

$$
\left[X^{k}+\left(\gamma F\left(\bar{X}^{k-1}\right)^{T}-X^{k-1}\right)\right]^{T} \cdot\left[X^{\prime}-X^{k}\right] \geq 0, \quad \forall X^{\prime} \in K .
$$

\section{Step 3: Convergence verification}

If $\left|X^{k}-X^{k-1}\right| \leq \varepsilon$, for $\varepsilon>0$, a prespecified tolerance, then stop; otherwise, set $k:=k+1$ and go to step 1 .

We now discuss the above algorithm more fully. We first recall the definition of the projection of $x$, on the closed convex set $K$, with respect to the Euclidean norm, and denoted by $P_{K} x$, as

$$
y=P_{K} x=\underset{z \in K}{\arg \min }\|x-z\| .
$$

In particular, we note that (cf. theorem 1.2 in Nagurney [9]) $\bar{X}^{k-1}$ generated by the modified projection method as the solution to the variational inequality subproblem (27) is actually the projection of $X^{k-1}-\gamma F\left(X^{k-1}\right)^{T}$ on the closed convex set $K$, where here $K$ is simply the nonnegative orthant. In other words,

$$
\bar{X}^{k-1}=P_{K}\left[X^{k-1}-\gamma F\left(X^{k-1}\right)^{T}\right] .
$$

Similarly, $X^{k}$ generated by the solution to variational inequality subproblem (28) is the projection of $X^{k}-\gamma F\left(\bar{X}^{k-1}\right)^{T}$ on the nonnegative orthant, that is,

$$
X^{k}=P_{K}\left[X^{k-1}-\gamma F\left(\bar{X}^{k-1}\right)^{T}\right] .
$$


Since the feasible set here is of box type, the above projections immediately decompose across the coordinates of the feasible set. In fact, the solution of each of the variables encountered in (27) and (28) amounts to projecting onto $\mathbb{R}_{+}$separately.

Consequently, we can provide closed-form expressions for the solution of problems (27) and (28). In particular, we have that (27) can be solved as: For all supply markets $i, i=1, \ldots, m$, set

$$
\bar{\pi}_{i}^{k-1}=\max \left\{0, \gamma\left(-s_{i}\left(\pi^{k-1}\right)+\sum_{k} Q_{i j}^{k-1}\right)+\pi_{i}^{k-1}\right\} .
$$

For all supply and demand market pairs $(i, j), i=1, \ldots, m ; j=1, \ldots, n$, set

$$
\bar{Q}_{i j}^{k-1}=\max \left\{0, \gamma\left(\left(-\pi_{i}^{k-1}-c_{i j}\left(Q^{k-1}\right)\right)\left(1+\tau_{i j}\right)+\rho_{j}^{k-1}\right)+Q_{i j}^{k-1}\right\} .
$$

Finally, for all demand markets $j, j=1, \ldots, n$, set

$$
\bar{\rho}_{j}^{k-1}=\max \left\{0, \gamma\left(d_{j}\left(\rho^{k-1}\right)-\sum_{i} Q_{i j}^{k-1}\right)+\rho_{j}^{k-1}\right\} .
$$

On the other hand, (28) can be solved explicitly in closed form as: For all supply markets $i, i=1, \ldots, m$, set

$$
\pi_{i}^{k}=\max \left\{0, \gamma\left(-s_{i}\left(\bar{\pi}^{k-1}\right)+\sum_{k} \bar{Q}_{i j}^{k-1}\right)+\pi_{i}^{k-1}\right\} .
$$

For all supply and demand market pairs $(i, j), i=1, \ldots, m ; j=1, \ldots, n$, set

$$
Q_{i j}^{k}=\max \left\{0, \gamma\left(\left(-\bar{\pi}_{i}^{k-1}-c_{i j}\left(\bar{Q}^{k-1}\right)\right)\left(1+\tau_{i j}\right)+\bar{\rho}_{j}^{k-1}\right)+Q_{i j}^{k-1}\right\},
$$

and, finally, for all demand markets $j, j=1, \ldots, n$, set

$$
\rho_{j}^{k}=\max \left\{0, \gamma\left(d_{j}\left(\bar{\rho}^{k-1}\right)-\sum_{i} \bar{Q}_{i j}^{k-1}\right)+\rho_{j}^{k-1}\right\} .
$$

In view of expressions (32)-(34) and (35) -(37), one sees that all of the $m$ supply price subproblems, the $m n$ commodity shipment subproblems, and the $n$ demand price subproblems can be solved simultaneously at each iteration. Hence, an "ideal" computer architecture for the solution of such problems may be one in which there are as many processors as there are pairs of markets. We investigate this issue in the subsequent section.

The convergence results are now given. 
Theorem 2 Assume that $s(\pi)$ and $-d(\rho)$ are each strongly monotone in $\pi$ and $\rho$, respectively, and that $c(Q)$ is strongly monotone in $Q$, and that all these functions have bounded first-order derivatives. Also, assume that the tariff rates $\tau_{i j}$ are finite for all $i, j$. Then the modified projection method converges to the solution of variational inequality (8), provided that a solution exists.

Proof As established in Korpelevich [8], the modified projection method is guaranteed to converge if the function $F(\cdot)$ that enters the variational inequality problem is monotone and Lipschitz continuous. Monotonicity follows from lemma 3, and Lipschitz continuity from lemma 1 , under the above assumptions.

We note that the projection method (cf. Dafermos [5], Bertsekas and Tsitsiklis [1]), which does not require the adaptation step 2 in the modified projection method, nevertheless requires stronger conditions for convergence, in particular, strong monotonicity of the function $F$, rather than monotonicity.

In Nagurney et al. [10], convergence results are provided for the modified projection method as applied to the spatial equilibrium model in quantity variables with ad valorem tariffs and with fixed transaction costs. As noted earlier, the number of variables that need to be computed in quantity models is less than the number arising in price and quantity models, in particular, a total of $m n$ commodity shipment variables rather than a total of $m+m n+n$ variables. Hence, the modified projection method in the context of quantity models yields only a single set of subproblems at each step, in contrast to the three sets of subproblems at each step (cf. (32)-(34) and (35)-(37)).

\section{Massively parallel implementation of the algorithm with numerical results}

In this section, we describe the massively parallel implementation of the modified projection method, defined by expressions (27) and (28), for the computation of the price and quantity formulation of the spatial equilibrium problem with ad valorem tariffs, which takes on the explicit formulas given by (32)-(34) and (35)-(37). We then present numerical results for large-scale problems. We consider problems that include both nonlinear and asymmetric functions. The language used in the implementation was CM FORTRAN and the architectures selected for the computations were the Thinking Machine's CM-2 machine and the CM-5, also commonly referred to as the Connection Machines.

The CM-2 is a distributed memory SIMD (Single-Instruction Multiple Data) massively parallel processing system. The mode of computation is data-level parallelism, that is, all processors execute identical operations. The CM-2 system utilized allowed for access of $8 \mathrm{~K}(8,192), 16 \mathrm{~K}(16,384)$, or $32 \mathrm{~K}(32,768)$ processors in its configuration. 
The CM-5, on the other hand, is an example of an MIMD (Multiple-Instruction Multiple Data) architecture. It consists of processing nodes that are SPARC processors, each of which has four proprietary attached vector units. A group of nodes under the control of a single processor is called a partition. The system that we utilized allowed for access of 32 nodes, 64 nodes, 256 nodes, or 512 nodes.

\subsection{Implementation}

We now discuss the implementation of the modified projection method in CM FORTRAN. In particular, we considered a linear, asymmetric supply function for each supply market $i$, of the form

$$
s_{i}(\pi)=\sum_{j} \frac{1}{r_{i j}} \pi_{j}-\frac{t_{i}}{r_{i i}},
$$

and a linear asymmetric demand function for each demand market $j$, of the form

$$
d_{j}(\rho)=-\sum_{k} \frac{1}{m_{j k}} \rho_{k}+\frac{q_{j}}{m_{j j}} .
$$

The transaction cost function associated with each market pair $(i, j)$ was of the form

$$
c_{i j}\left(Q_{i j}\right)=\beta_{i j} Q_{i j}^{2}+g_{i j} Q_{i j}+h_{i j}
$$

We selected such supply and demand price function structures, since much of the previous literature considered linear functions (albeit typically symmetric functions, which we did not need to assume). On the other hand, we included nonlinearities in the unit transaction cost functions to demonstrate the performance of the algorithm on problems with nonlinearities.

The CM FORTRAN code for the implementation of the modified projection method for this model consisted of an input and setup routine and a computation routine to implement the iterative steps (32)-(34) and (35)-(37). The critical feature in the design of the program was the construction of the data structures to take advantage of the data level parallelism and computation. We, hence, defined arrays $G, Q$, and $H$, each of dimension $m \times n$, to store the elements $\left\{g_{i j}\right\},\left\{Q_{i j}\right\}$ at each iteration, and $\left\{h_{i j}\right\}$, respectively. Also, we constructed $m \times n$ dimensional arrays SPRICE and DPRICE, with each element of row $i$ of SPRICE containing $\pi_{i}$, and with each element of column $j$ of DPRICE containing $\rho_{j}$, at each particular iteration. The arrays SPR and DPR stored the new values of $\pi$ and $\rho$, respectively, at a particular iteration.

The matrix of supply coefficient $\left\{1 / r_{i j}\right\}$ was stored in an $m \times m$ array $1 / R R$, and the matrix of demand coefficients $\left\{-m_{j k}\right\}$ was stored in an $n \times n$ array $1 /(-M M)$. To compute the supply function values, we used the spread command to spread the supplies and then multiplied the resulting matrix with the $1 / R R$ matrix. We subsequently used the sum command to add the elements of each row. Finally, this vector 
was added to the vector $t / R$ containing the fixed supply price terms (cf. (38)). The demand prices were obtained in an analogous fashion. With regard to the transaction cost functions, an additional array G2 was introduced to store the coefficients $\left\{\beta_{i j}\right\}$.

We constructed the array "tau" to store the tariff coefficients, with the $(i, j)$ th component containing $\tau_{i j}$.

The algorithm was initialized, for all the examples, with $Q^{0}=0, \pi^{0}=0$, and $\rho^{0}=0$, with the convergence tolerance $\varepsilon$ set to 0.001 . Only this starting point was used. The parameter $\gamma$ was set to 0.001 for all the computations.

For the interested reader, we now present the critical steps in the CM FORTRAN computation section.

\section{FORTRAN implementation}

Do while (err.ge..001.or.err1..ge..001..or.err2.ge..001)

\section{c Initialization}

Step 1. $\mathrm{QO}(:,:)=\mathrm{Q}(:,:)$

$\operatorname{SPRO}(:)=\operatorname{SPR}(:)$

$\operatorname{DPRO}(:)=\mathrm{DPR}(:)$

\section{c Construction and computation}

Step 2. $\operatorname{SPRICE}(:,:)=$ spread $(\operatorname{SPRO}(:), \operatorname{dim}=2, \mathrm{ncopies}=\mathrm{n})$

$\operatorname{DPRICE}(:,:)=\operatorname{spread}(\mathrm{DPRO}(:), \operatorname{dim}=1, \mathrm{ncopies}=\mathrm{m})$

Step 3. $\operatorname{temp}(:,:)=\mathrm{QO}(:,:)+\gamma *$

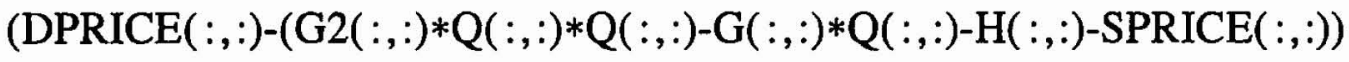
$*(1+\operatorname{tau}))$

Step 4. $Q(:,:)=\operatorname{temp}(:,:)$

Step 5. where (temp(:,:).lt.0.) $\mathrm{Q}(:,:)=0$.

Step 6. $\operatorname{stemp}(:)=\operatorname{sum}(\mathrm{QO}(:,:), 2)$

$\operatorname{stemp1}(:,:)=\operatorname{spread}(\operatorname{stemp}(:), \operatorname{dim}=1$, ncopies $=m)$

$\operatorname{stemp} 2(:,:)=\frac{1}{R R}(:,:) * \operatorname{stemp} 1(:,:)$

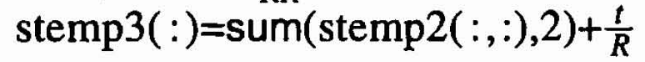

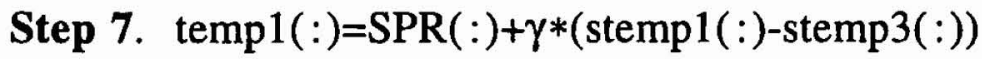

Step 8. where(temp1(:).1t.0.) $\operatorname{SPRO}(:)=0$.

Step 9. Compute the demand counterparts

\section{c Adaptation}

(follow as above with $\mathrm{Q}(:,:)+$ in step 3 replaced by $\mathrm{QO}(:,:)+$, $\operatorname{SPR}(:,:)$ in step 7 replaced by $\operatorname{SPRO}(:,:)$, etc.) 


\section{c Convergence verification}

Step 10. err=maxval $(\operatorname{abs}(\mathrm{Q}-\mathrm{QO}))$

err $1=\operatorname{maxval}($ abs(SPR-SPRO) $)$

err2 $=(\operatorname{abs}($ SPR-DPRO $))$

end do

For example, expression (33) of the algorithm corresponds to steps $3-5$ and expression (32) corresponds to steps 6-8.

We solved five problems, of dimensions $m \times n$, in particular, $100 \times 100,200 \times 200$, and so on, until $500 \times 500$. The problems considered here were large scale; example 1 had a total of 10,000 commodity shipment variables and 200 price variables, whereas example 5 had a total of 250,000 commodity shipment variables and 1,000 price variables.

The data (cf. (38), (39), and (40)) were generated randomly and uniformly in the ranges: $1 / r_{i i} \in[10,30],-t_{i} / r_{i i} \in[10,100],-m_{j j} \in[-5,-55], q_{j} / m_{j j} \in[50,5000]$, $\beta_{i j} \in[0.05,0.55], g_{i j} \in[2,60]$, and $h_{i j} \in[5,50]$, for all $i=1, \ldots, m ; j=1, \ldots, n$. The offdiagonal terms in the supply and demand price functions were generated to ensure strict diagonal dominance and, hence, the functions were strongly monotone and had bounded second-order derivatives, thus satisfying conditions for convergence of the modified projection method. Each demand and supply function had five terms.

The tariff rates, in turn, were generated randomly and uniformly in the ranges $\tau_{i j} \in[0,2]$, for all $i, j$. This range was selected to coincide with the ranges commonly used in practice, and in recently negotiated trade agreements.

The numerical results are reported in table 1 for the problems run on the CM-2 architecture and in table 2 for the same problems run on the CM-5. In particular, we report the CPU times, that is, the CM-2 times and the CM-5 times required for convergence of the algorithm for each example to the tolerance set previously.

The first example in this set required 1,243 iterations for convergence, the second example required 1,239 iterations, the third example 3,261 iterations, the fourth 1,904 iterations, and the fifth 1,456 iterations for convergence. The fourth example required less time than the third example, although it was larger, due to the fewer number of iterations required.

We also measured the computational accuracy of the modified projection method in terms of the average error, where

$$
\text { Average Error }=\frac{100}{p} \sum_{i j} \frac{\left|\left(\pi_{i}+c_{i j}\right)\left(1+\tau_{i j}\right)-\rho_{j}\right|}{\left(\pi_{i}+c_{i j}\right)\left(1+\tau_{i j}\right)},
$$

where $p=$ the number of supply and demand market pairs $(i, j)$ such that $Q_{i j}>0$, and the maximum error, where

$$
\text { Maximum Error }=\max _{i j} \frac{100\left|\left(\pi_{i}+c_{i j}\right)\left(1+\tau_{i j}\right)-\rho_{j}\right|}{\left(\pi_{i}+c_{i j}\right)\left(1+\tau_{i j}\right)},
$$


Table 1

CM-2 times for spatial price equilibrium problems with ad valorem tariffs, price and quantity formulation.

\begin{tabular}{cccccc}
\hline Example & $\begin{array}{c}\text { No. of supply } \\
\text { markets }\end{array}$ & $\begin{array}{c}\text { No. of demand } \\
\text { markets }\end{array}$ & \multicolumn{3}{c}{ CM-2 time (sec.) } \\
\hline 1 & 100 & 100 & 26.61 & 19.04 & 15.74 \\
2 & 200 & 200 & 73.01 & 34.43 & 25.28 \\
3 & 300 & 300 & 163.81 & 98.55 & 70.48 \\
4 & 400 & 400 & 140.62 & 83.64 & 58.45 \\
5 & 500 & 500 & 178.97 & 135.65 & 77.05 \\
\hline
\end{tabular}

Table 2

CM-5 times for spatial price equilibrium problems with ad valorem tariffs, price and quantity formulation.

\begin{tabular}{ccccc}
\hline Example & $\begin{array}{c}\text { No. of supply } \\
\text { markets }\end{array}$ & $\begin{array}{c}\text { No. of demand } \\
\text { markets }\end{array}$ & \multicolumn{2}{c}{ CM-5 time (sec.) } \\
256 nodes
\end{tabular}

for all $(i, j)$ such that $Q_{i j}>0$. Such measures of accuracy have been used by, among others, Nagurney et al. $[10,11]$.

The average error for example 1 was 0.006 and the maximum error was 1.406 . The percentage of positive commodity shipments was $47.35 \%$. For example 2 , the average error was 0.007 and the maximum was 2.183 , with $33 \%$ of the commodity shipments being positive. Example 3 has an average error of 0.000 and a maximum of 0.009 , with $27.10 \%$ positive shipments. Example 4 had $26.10 \%$ positive shipments, and average error of 0.001 and a maximum error of 0.703 . Finally, example 5 had $21.75 \%$ positive shipments at convergence, an average error of 0.004 and a maximum error of 5.681 .

We report the CPU (CM-5) times for the implementation of the algorithm on the CM-5 architecture in table 2.

These numerical results suggest that our implementation of the modified projection method for the computation of spatial price equilibria in the presence of ad valorem tariffs was efficient. Moreover, all the CPU times on only 64 nodes of the CM-5 were less than the CPU times on the CM-2. Finally, the above results strongly suggest that the algorithm, which is a massively parallel algorithm, should be imple- 
mented on a massively parallel architecture. We did not implement the algorithm on a serial architecture in view of the computational comparisons conducted in Nagurney et al. [11], wherein a massively parallel implementation of an Euler-type method was used to solve spatial price equilibrium problems formulated as dynamical systems on the CM-2. In addition, the results in Nagurney et al. [10] strongly suggest that for spatial price equilibrium problems in quantity variables and with ad valorem tariffs, the modified projection method should clearly be implemented on a massively parallel architecture for problems with more than 10,000 commodity shipment variables.

In conclusion, the above numerical results demonstrate that problems as large as those containing 251,000 variables can now be solved in only seconds of CPU time when the appropriate algorithm is matched for a given problem and architecture. These computational results, therefore, show that one can evaluate alternative policy scenarios in the form of alternative tariffs in a timely manner. Moreover, the result clearly show that the CPU times obtained in the CM-5 were consistently superior to those obtained on the CM-2. Indeed, the largest problems, examples 3, 4, and 5, were solved in, on the average, half the time on the CM-5 with 256 nodes that was required to solve the same problem in the full configuration of the CM-2 with $32 \mathrm{~K}$ processors. This is not surprising given the two architectures, but illuminating nonetheless.

\section{Summary and conclusions}

In this paper, we developed a new spatial equilibrium model in price and quantity variables and with ad valorem tariffs. Ad valorem tariffs are a commonly used policy instrument by many nations and a common feature of many trade negotiations. Nevertheless, to date, spatial market methods with ad valorem tariffs have made such simplifying assumptions as linear and, oftentimes, separable functions. Moreover, it is spatial price equilibrium models in quantity variables, with or without ad valorem tariffs, even in the general variational inequality framework that have received more attention in the literature (cf. Nagurney et al. [10]). This may be due, in part, to the fact that because there are fewer variables in quantity models, the algorithmic development for such problems may be less complex.

Here, we proposed the modified projection method for the computation of the equilibrium price and commodity shipment pattern, and provided convergence results. The realization of the algorithm in the context of our model yields three sets of subproblems, in supply price, commodity shipment, and demand price variables, each of which, in turn, can be solved explicitly in closed form. In addition, all of the subproblems, the number of which is equal to the number of supply markets plus the number of demand markets plus the number of commodity shipments, can be solved simultaneously. Hence, the algorithm, for this problem, at least in principle, can be implemented on massively parallel architectures.

We then outlined the implementation of the algorithm in CM FORTRAN and provided numerical results for large-scale problems with as many as 251,000 variables. 
The computational comparisons were conducted on the massively parallel architectures, the SIMD Thinking Machines CM-2 and the MIMD Thinking Machines CM-5, known as the Connection Machines. The numerical results demonstrate the efficiency of the implementation of the algorithm and the importance of matching algorithm to application to computational platform. Indeed, the largest problems solved required only seconds of CPU time, thus allowing for the evaluation of alternative policy scenarios in a timely fashion.

Finally, we note that this research complements the recent work of Nagurney et al. [11], where the Euler method was implemented on the CM-2 for the formulation of spatial price equilibrium problems in quantity variables and as dynamical systems, and the work of Nagurney et al. [10], where the modified projection method was implemented on the CM-2 for the tariff model, but in quantity variables only.

\section{Acknowledgements}

The first author's research was supported by the National Science Foundation under grant DMS 9024071 under the Faculty Awards for Women program. This support is gratefully acknowledged. This research was conducted at the National Center for Supercomputer Applications at the University of Illinois at Urbana. The authors would like to thank the two anonymous referees for many useful suggestions and comments that improved the presentation of this work.

\section{References}

[1] D.P. Bertsekas and J.N. Tsitsiklis, Parallel and Distributed Computation, Prentice-Hall, Englewood Cliffs, NJ, 1989.

[2] C.C. Chang, B.A. McCarl, J.W. Mjelde and J.W. Richardson, Sectoral implications of farm program modifications, American Journal of Agricultural Economics 74 (1991).

[3] G.L. Cramer, E.J. Wailes, J.M. Goroski and S.S. Phillips, The impact of liberalizing trade on the world rice market: A spatial model including rice quality, Arkansas Agricultural Experiment Station, Fayetteville, AR, 1991.

[4] S. Dafermos, Traffic equilibrium and variational inequalities, Transportation Science 14 (1980).

[5] S. Dafermos, An iterative scheme for variational inequalities, Mathematical Programming 26 (1983).

[6] S. Dafermos and S. McKelvey, Partitionable variational inequalities with applications to network and economic equilibrium, Journal of Optimization Theory and its Applications 73 (1992).

[7] F.D. Holland and J.A. Sharples, World wheat trade: Implications for U.S. exports, Staff Paper No. 84-20, Department of Agricultural Economics, Purdue University, West Lafayette, IN, 1984.

[8] G.M. Korpelevich, The extragradient method for finding saddle points and other problems, Ekon. i Mat. Metody, translated as Matekon 13 (1976).

[9] A. Nagurney, Network Economics: A Variational Inequality Approach, Kluwer Academic, Boston, MA, 1993.

[10] A. Nagurney, C.F. Nicholson and P.M. Bishop, Spatial price equilibrium models with discriminatory ad valorem tariffs: Formulation and comparative computation using variational inequalities, in: Recent Advances in Spatial Equilibrium Modelling: Methodology and Applications, J.C.J.M. van den Bergh, P. Nijkamp and P. Rietveld, eds., Springer, Berlin, 1995, to appear. 
[11] A. Nagurney, T. Takayama and D. Zhang, Massively parallel computation of spatial price equilibria as dynamical systems, Journal of Economic Dynamics and Control 18(1995).

[12] A. Nagurney, S. Thore and J. Pan, Spatial market policy modeling with goal targets, Operations Research 44(1996).

[13] A. Nagurney and L. Zhao, A network equilibrium formulation of market disequilibrium and variational inequalities, Networks 21 (1991).

[14] J.M. Ortega and W.C. Rheinboldt, Iterative Solution of Nonlinear Equations in Several Variables, Academic Press, New York 1970.

[15] P. Samuelson, Spatial price equilibrium and linear programming, American Economic Review 42 (1952).

[16] G. Strang, Introduction to Linear Algebra, Wellesley - Cambridge Press, Wellesley, MA, 1993.

[17] T. Takayama and G.G. Judge, An intertemporal price equilibrium model, Journal of Farm Economics 46 (1964).

[18] T. Takayama and G.G. Judge, Spatial and Temporal Price and Allocation Models, North-Holland, Amsterdam, 1971.

[19] G.L. Thompson and S. Thore, Economic disequilibrium by mathematical programming, Journal of Optimization Theory and its Applications 72 (1991).

[20] S. Thore, Spatial disequilibrium, Journal of Regional Science 26 (1986).

[21] S. Thore, Economic Logistics, Quorum Books, New York, 1991.

[22] S. Thore, A. Nagurney and J. Pan, Generalized goal programming and variational inequalities, Operations Research Letters 12 (1992). 